\title{
Le plurilinguisme trouve-t-il sa traduction réelle dans la formation professionnelle des enseignants du niveau primaire?
}

\section{Anna Butašová}

\section{OpenEdition}

\section{Journals}

Édition électronique

URL : http://journals.openedition.org/trema/3261

DOI : $10.4000 /$ trema.3261

ISSN : 2107-0997

Éditeur

Faculté d'Éducation de l'université de Montpellier

Édition imprimée

Date de publication : 1 novembre 2014

Pagination : $154-161$

ISSN : 1167-315X

Référence électronique

Anna Butašová, « Le plurilinguisme trouve-t-il sa traduction réelle dans la formation professionnelle des enseignants du niveau primaire? », Tréma [En ligne], 42 | 2014, mis en ligne le 16 juin 2015, consulté le 05 mai 2019. URL : http://journals.openedition.org/trema/3261 ; DOI : 10.4000/ trema.3261

Ce document a été généré automatiquement le 5 mai 2019.

Trema 


\title{
Le plurilinguisme trouve-t-il sa traduction réelle dans la formation professionnelle des enseignants $d u$ niveau primaire?
}

\author{
Anna Butašová
}

\section{Le plurilinguisme dans le contexte slovaque}

1 La problématique du plurilinguisme résonne dans toute l'Europe, la Slovaquie n'y fait pas exception. Il n'est pas exceptionnel non plus que le système éducatif slovaque connaisse des réformes, y compris celle en cours, visant à optimiser le système éducatif face aux exigences de sa modernisation. En Europe centrale, les débuts de la réforme ou bien des réformes datent des années $90 \mathrm{du}$ siècle précédent. Mais surtout, ces réformes restent inachevées et continuent à être mises en œuvre dans les conditions du fonctionnement normal du système éducatif. Pour la plupart des cas, elles réagissent aux problèmes engendrés par une vague d'évaluations de compétences au niveau international (PISA, TIMS, etc.) ou encore elles répondent aux résultats des évaluations mises en place au niveau national. Pour les concepteurs des programmes d'enseignement (terme qui, en Slovaquie, s'est substitué de manière stable au terme de curriculum), cela signifie la difficulté d'intégrer des paramètres en cours de changements permanents et de gérer très souvent des modifications aléatoires pouvant affaiblir des liens entre les compétences transversales. En ce qui concerne les langues étrangères, cette situation est d'autant plus compliquée que la mise en œuvre des recommandations élaborées par le Conseil de l'Europe, par la Commission européenne et adoptées par la République slovaque, en sa qualité d'État membre du Conseil européen et de l'Union européenne, reste soumise à des pressions, des représentations politiques au pouvoir et au financement très faible du système éducatif. Dès à présent, on peut constater qu'avec le financement maintenu au 
niveau actuel il sera impossible de relever le défi des innovations souhaitées. Selon les sources de l'OCDE datant de 2010, le système éducatif slovaque n'a représenté que 4,70\% du PIB.

2 Au sein de l'éducation nationale slovaque, la langue étrangère est considérée comme une matière importante, traditionnellement se trouvant au centre de l'intérêt d'intérêt des apprenants, des parents et du grand public. La République slovaque, du point de vue de son évolution historique, est un pays où le multilinguisme et le plurilinguisme sont fort présents. La présence des minorités sur le territoire de Slovaquie après la première guerre mondiale menait naturellement à une diversité linguistique et en même temps à une diversité de cultures et d'influences culturelles. En 1921, la Slovaquie compte environ 140000 Allemands, 640000 Hongrois, 85000 Ruthènes, 70000 Juifs et 45000 représentants d'autres minorités. A l'époque, la Slovaquie comptait 2 millions d'habitants (Slovenský náučný slovník [Dictionnaire encyclopédique slovaque], 1932). Il en découle clairement qu'au moment de la formation des structures européennes, au début du XXe siècle, le plurilinguisme est un élément constitutif du savoir-être, déraciné progressivement plus tard des structures et des systèmes sociaux.

3 Ces références historiques, illustrant bien la situation sociale en Slovaquie au début du XXe siècle, nous amènent à nous poser la question suivante: quelle approche du plurilinguisme notre système éducatif devrait-il illustrer? Quelles sont l'étendue et la force des de comportements habituels dans un milieu où comprendre et se faire comprendre en plusieurs langues était appréhendé comme une compétence naturelle? $\mathrm{Au}$ milieu des années 50, Bratislava est réputée être trilingue grâce à sa position géographique aux frontières autrichienne et hongroise.

En Slovaquie, les résidus du plurilinguisme restent encore bien ancrés. Du point de vue anthropologique, il s'agit du plurilinguisme naturel connaissant une évolution liée aux étapes de l'évolution de la société ; selon le cas, l'essor du plurilinguisme était plus ou moins fort, tantôt stimulé, tantôt supprimé par les différentes politiques de l'État. À présent, la République slovaque est pays signataire de toutes les chartes et recommandations des institutions internationales, notamment du Conseil de l'Europe, visant les droits des minorités ainsi que le droit à l'utilisation de la langue de la minorité dans des situations formelles. Les politiques éducatives slovaques intègrent un réseau d'écoles dont la langue d'enseignement est la langue d'une minorité, qu'il s'agisse du hongrois, de l'allemand ou du ruthène. Toutefois, le problème de scolarisation des enfants roms reste ouvert. Pour ces enfants, la difficulté est double: d'une part, l'utilisation de leur langue est liée à un environnement défavorisé et peu stimulant de la communauté rom; d'autre part, cette dernière alimente les débats et la recherche constante de solutions. Par ailleurs, la Slovaquie adhère aux idées du plurilinguisme puisqu'elle respecte toutes les langues et les reconnaît, à la fois pour la scolarisation et pour l'utilisation en situation de communication officielle. Pourtant, l'utilisation d'une autre langue que celle de la population majoritaire peut susciter, dans certaines interactions, des réactions qui ne sont pas toujours positives. La perception d'une langue est davantage liée à des facteurs affectifs que rationnels. Les décisions politiques concernant l'utilisation et l'apprentissage des langues ainsi que la manière de les présenter peuvent largement contribuer à modifier cette perception. Lors du dernier recensement, 80,7\% des habitants se sont déclarés de nationalité slovaque, $8,5 \%$ de nationalité hongroise et $2 \%$ de nationalité rom. Le pourcentage des autres nationalités n'est pas connu, elles sont néanmoins assez nombreuses. Ce sont notamment les nationalités tchèque, ruthène, 
polonaise, ukrainienne, serbe, croate, juive, russe, bulgare (Štatistický úrad [Agence nationale des statistiques], 2012).

5 La diversité linguistique en Slovaquie se trouve complétée encore par les patois, si différents qu'ils peuvent devenir incompréhensibles pour l'habitant d'une autre région. Les patois sont parlés au niveau local en situation de communication familière. L'utilisation des patois en situation de communication officielle est perçue comme inadaptée et indécente.

\section{Variabilité dans l'enseignement des langues étrangères et problématique de la formation professionnelle des enseignants du niveau CITE 1}

6 Certaines mesures législatives en faveur du soutien au plurilinguisme ont été adoptées dans le cadre des politiques linguistiques et éducatives. Parmi ces dispositions figurent également celles qui déclarent pour objectif de l'enseignement des langues étrangères la maîtrise de deux langues étrangères à un niveau de compétences communicatives défini à la fin des études secondaires. Selon le Cadre européen commun de référence pour les langues, le niveau requis pour la première langue étrangère est le niveau B2, pour la deuxième langue étrangère le niveau A2-B1. La loi sur l'éducation (Loi n 245/2008 JO aux termes des dispositions ultérieures) introduit, à partir de 2011, la langue anglaise comme langue étrangère obligatoire durant les études, la deuxième langue étrangère pouvant être choisie parmi les langues enseignées par l'école. L'école est tenue de garantir la qualité de l'enseignement des langues étrangères conformément au paragraphe 4 de la loi précitée qui définit le contenu et les objectifs de l'apprentissage comme il suit :

L'objectif de l'éducation et de l'enseignement est de permettre à l'enfant ou à l'élève

1. d'accéder à une formation conformément à la loi ;

2. d'acquérir des compétences, notamment dans les domaines suivants: communication, production orale et écrite, utilisation des technologies de l'information et de la communication, communication en langue officielle, en langue maternelle et en langue étrangère, mathématiques, sciences naturelles, techniques et technologies, apprendre à apprendre tout au long de la vie, les compétences sociales et civiques, les compétences d'entreprise et les compétences culturelles ;

3. de maîtriser la langue anglaise et au minimum une autre langue ; être capable de les utiliser.

7 L'actuelle interprétation du paragraphe 4 par le Ministère de l'éducation nationale impose, à partir de l'année scolaire 2013/2014, la langue anglaise comme première langue étrangère sans qu'un budget particulier soit alloué à la mise en œuvre de cette décision.

En 2008, sur l'initiative du Ministère de l'éducation, un programme de réflexion sur la conception de l'enseignement des langues étrangères a été élaboré.

Ce programme (mis en place en 2009) visait à prendre en compte la situation sociale du pays dans l'introduction de l'apprentissage obligatoire d'une langue étrangère dans l'enseignement primaire, à partir de la troisième année de scolarisation obligatoire de l'enfant. Les analyses ont bien montré que, d'un côté, l'introduction d'une langue étrangère obligatoire depuis la troisième année correspond aux impératifs de l'époque actuelle et avant tout à la demande des parents d'enseigner une langue étrangère aux enfants depuis le jeune âge mais que, par ailleurs, l'enseignement manque d'enseignants qualifiés en langues étrangères, capables de dispenser un enseignement où la 
communication langagière ne serait pas l'objectif unique. Les spécialistes émettaient des avis alarmants: selon eux, les enseignants non qualifiés ne seraient pas en mesure d'assurer la mise en œuvre des approches modernes facilitant, dès les premiers contacts avec la langue, l'accès des apprenants aux nouvelles cultures et aux nouvelles connaissances.

Ces approches exigent que de multiples liens soient établis entre les matières enseignées au premier cycle de l'enseignement élémentaire. C'est au travers d'une langue étrangère que les apprenants devraient être capables de découvrir leur identité linguistique et de saisir les différences entre deux systèmes linguistiques.

11 Un enseignant non qualifié ne saura pas mettre au profit de l'enseignement l'altérité d'une communauté linguistique et culturelle. En 2010, avec l'entrée en vigueur de la loi fixant l'apprentissage obligatoire d'une langue étrangère depuis le premier cycle de scolarité, se pose le problème des enseignants non qualifiés. Non seulement ces enseignants ne possèdent aucune qualification les habilitant à enseigner une langue étrangère, mais ils ne maîtrisent aucune langue étrangère. Ainsi, depuis 2011, de nouvelles dispositions légales permettent à toute personne ayant passé son examen de baccalauréat ou un examen d'État de langue étrangère, d'enseigner cette matière au niveau élémentaire. Aucune formation pédagogique n'est exigée. Le seul élément positif est que ces dispositions sont prises pour une durée limitée.

Des aperçus sur la situation dans l'Union européenne montrent bien que plusieurs États ont été contraints de mettre en place des dispositifs similaires. Du point de vue des politiques linguistiques, la problématique prend une autre dimension.

13 Les indicateurs quantitatifs nous renseignent sur le fait que l'apprentissage des langues étrangères à un jeune âge est mis en place à travers toute l'Europe mais la qualité de cet apprentissage reste inconnue. Malgré cet état de fait contradictoire, les universités formant les pédagogues pour le niveau élémentaire ne réagissent pas et les didactiques des langues étrangères ne sont pas incorporées dans les programmes d'études des futurs maîtres d'école, ne serait-ce qu'au niveau des matières facultatives. Cette situation est due principalement au fait que la décision politique en faveur du soutien au plurilinguisme en République slovaque n'a été accompagnée d'aucune mesure concernant son financement. Avec le financement du système éducatif à 4,7\% du PIB dont seulement $0,7 \%$ est réservé à l'enseignement supérieur, on ne peut s'attendre à de grandes initiatives de la part des universités.

14 La formation universitaire des maîtres d'école est de 10 semestres qui couvrent deux cycles (l'un de 6 semestres, l'autre de 4 semestres) et elle est sanctionnée par un diplôme de master. Les diplômés sont considérés comme des pédagogues qualifiés de l'enseignement élémentaire.

15 La contradiction entre le contenu de la formation et la loi sur l'éducation définissant les objectifs de l'enseignement au niveau de CITE 0 à CITE 3 est évidente. Une matière obligatoire est introduite dans l'enseignement sans qu'aucune articulation avec la formation professionnelle des enseignants ne soit assurée. Cette anomalie éducative se répercute sur la qualité de l'enseignement. Le volume horaire hebdomadaire est un autre facteur aggravant. Etant donné que chaque école dispose d'un certain nombre d'heures «libres » pouvant être attribuées à une matière en vue de la renforcer, il arrive que le chef d'établissement décide, paradoxalement, de les attribuer à la langue étrangère. Ce que nous pouvons affirmer avec certitude, c'est qu'à la sortie du cycle élémentaire nous 
connaissons deux paramètres :

celui des performances se situant au niveau A1.1+ selon le Cadre européen commun pour les langues et celui du nombre d'heures hebdomadaires minimum.

En juin 2012, les apprenants du niveau élémentaire ont passé des tests d'anglais. Dans le cadre d'un projet de recherche, un test de niveau A1.1+ a été administré à un échantillon de 738 élèves de la région de Žilina. Le résultat obtenu par cet échantillon, choisi en fonction de plusieurs paramètres, peut être représentatif de la population des élèves du cycle élémentaire en République slovaque. La qualité de l'instrument de mesure a été évaluée par la Théorie classique du test ainsi que par les analyses psychométriques. Ainsi, la fidélité et la validité du test ont été assurées. Deux variantes (A, B) de test ont été élaborées pour trois compétences - listening, reading, writing. La variation de l'intervalle de réussite était de $64,26 \%$ à $71,70 \%$. Ces résultats témoignent d'un bon niveau, cependant ils ne fournissent aucun renseignement sur les attitudes des apprenants envers la matière ou encore envers la langue enseignée. Ce test n'évalue pas la compétence plurilingue bien que cette compétence, selon les études récentes du Conseil de l'Europe, soit une composante importante des curriculums modernes.

\section{Projet national de la formation continue des enseignants du niveau CITE 1}

17 En 2008, le Ministère de l'éducation nationale de la République slovaque donne son autorisation à la mise en œuvre du projet de formation continue des enseignants de langues étrangères au niveau élémentaire, sous condition que ce projet de cinq ans serve de pont entre les premiers élémentaristes diplômés et formés pour l'enseignement des langues étrangères et ceux formés dans le cadre du projet de la formation continue, l'objectif de celle-ci étant d'élargir la qualification pour les langues étrangères des enseignants en postes. Alors que la définition du projet était bien claire, la formation initiale des futurs enseignants élémentaristes n'a pas changé. Ainsi, une contradiction profonde existe entre l'obligation d'enseigner les langues étrangères depuis la troisième année de scolarité fixée par la loi et l'actuelle formation universitaire des enseignants du niveau élémentaire.

Le projet intitulé «Formation des enseignants des écoles primaires dans le domaine des langues étrangères en liaison avec la Conception de l'enseignement des langues étrangères dans les écoles primaires et secondaires » était un projet ambitieux. Soutenu par l'Union européenne, il avait pour objectifs d'élargir la qualification des enseignants, de promouvoir le plurilinguisme - ce projet a été mené pour six langues (anglaise, allemande, française, russe, espagnole et italienne) parmi lesquelles les enseignants pouvaient choisir - et de préparer les enseignants à réfléchir sur les enjeux du développement de la compétence plurilingue des élèves. Huit universités slovaques ont participé à ce projet d'envergure et leurs programmes d'études se focalisaient sur la mise en place de fondements pour une approche interculturelle, accompagnée de cours de méthodologie et de didactique des langues. Ces cours ont permis de procéder à des analyses de situations réelles liées aux processus d'enseignement et d'apprentissage d'une langue étrangère.

19 En République slovaque, les enseignants ne disposent pas de Portfolio validé pour les jeunes élèves. Ainsi l'absence de cet outil important pour la formation plurilingue se fait 
sentir. D'autres outils pour la mise en œuvre du plurilinguisme dans les écoles, élaborés par le Conseil de l'Europe, ont été utilisés. Les formations ont permis aux enseignantsapprenants de saisir le concept du plurilinguisme à l'école ainsi que les principes didactiques qui le font vivre et permettent de le développer. À travers une pédagogie de projet, les enseignants cherchaient les possibilités de la mise en valeur des relations entre la langue étrangère et les autres matières enseignées dans leurs écoles, ou les possibilités d'orienter les comportements et les attitudes des apprenants vers une plus grande tolérance et ouverture à d'autres nations et langues mais aussi à l'autoévaluation et la prise en compte de leur propre compétence plurilingue. Plusieurs de ces réflexions se sont traduites en projets de qualité. Nous avons pu constater que l'éducation est un espace naturellement ouvert à la pluralité où les idées de plurilinguisme peuvent être mises en œuvre sans problèmes et peut-être même sans une préparation spécifique ou trop intense. Cependant, il importe qu'un discours social continu portant sur ce sujet soit tenu tant au sein des établissements de formation professionnelle des enseignants que dans le milieu éducatif. Les politiques linguistiques et éducatives devront créer les conditions favorables au développement d'une société plurilingue et pluriculturelle.

On peut penser qu'avec l'arrivée à terme du projet, en juin 2013, la République slovaque pourrait disposer, à partir de l'année scolaire 2013/2014, de 3287 enseignants qualifiés au niveau élémentaire dont $85 \%$ en langue anglaise, $11,7 \%$ en langue allemande, $2,2 \%$ en langue russe et $1,1 \%$ en langues romanes (par ordre d'importance : français, espagnol, italien). Cela signifie qu'en 2013/2014, il y aurait dans les écoles slovaques 2803 enseignants d'anglais et 484 enseignants en langues étrangères autres (par ordre d'importance : allemand, russe, français, italien, espagnol). À première vue, on pourrait avancer qu'avec 3287 enseignants qualifiés, nous avons résolu le problème de l'enseignement qualifié des langues étrangères au niveau élémentaire à 100\%. Etant donné l'âge des enseignants, cette situation pourrait rester satisfaisante pendant environ 8 ans. Avec les départs à la retraite, elle commencerait à se dégrader. En 2010/2011, 140 053 élèves du primaire apprenaient une langue étrangère, soit $70,2 \%$ des apprenants du total des élèves alors qu'en 2006/2007, ils n'étaient que 107813 , soit la moitié du total des élèves. Nous constatons une progression de 32240 apprenants d'une langue étrangère, soit une croissance de $29,9 \%$.

\section{Conclusion}

21 Les systèmes éducatifs des Etats démocratiques sont aujourd'hui affectés par différentes tensions; toutefois, ils affirment des objectifs ambitieux en vue de préparer les enfants à s'intégrer dans une société globale et d'assurer la possibilité de leur développement intellectuel individuel. L'intention de sauvegarder les principes du développement des différentes cultures, y compris celles qui sont minoritaires, est partout observée. Dès son entrée dans le système éducatif, l'enfant est soumis aux différents systèmes hiérarchiques : à la hiérarchie sociale (la récession actuelle fait que le nombre d'enfants des communautés pauvres augmente); à la hiérarchie linguistique (les migrations renforcent les différences établies en fonction des tendances unificatrices de presque tous les systèmes éducatifs démocratiques où l'unification s'opère selon le modèle de correspondance entre la langue d'enseignement et la langue officielle et ne tient pas compte des langues maternelles); enfin, à d'autres hiérarchies encore, selon les spécificités des pays. Il en résulte un intérêt croissant pour le développement de la 
compétence plurilingue des apprenants. Celle-ci ouvre la voie de la valorisation des racines linguistiques et culturelles de l'individu, et facilite la prise en compte de la différence dans l'unification. La problématique du plurilinguisme se voit souvent réduite à l'offre des langues étrangères enseignées. Sans sous-estimer l'importance du choix des langues, nous voulions mettre ici l'accent sur le plurilinguisme au sens plus large du terme. La compétence plurilingue devrait se projeter dans les relations et contextes interdisciplinaires au sein desquels l'enfant est placé. La réalisation de cette tâche incombe aux concepteurs des programmes d'études qui devraient, malgré les traditions universitaires, prendre davantage en considération la réalité actuelle. Par notre projet, nous avons essayé d'y contribuer dans l'espoir que les futures générations puissent en bénéficier.

\section{RÉSUMÉS}

Les politiques linguistiques, en République slovaque, reflètent la situation réelle à deux niveaux. Le premier niveau est représenté par le soutien des conditions favorables à la société plurilingue et pluriculturelle issue de l'histoire. Ce soutien se traduit par un dispositif légal permettant d'utiliser les langues des minorités comme la langue d'enseignement et la langue de communication officielle à côté de la langue nationale. Le second niveau est représenté par les politiques linguistiques dans le domaine de l'enseignement des langues étrangères. Ici, la situation ne trouve pas d'équilibre, elle change trop souvent et la formation professionnelle des enseignants du niveau CITE 1 (école élémentaire) reste sans solution.

The existing situation in the language policy in the Slovak Republic is reflected at two levels. The first level is the support of good conditions for the plurilingual and pluricultural society which was rooted historically. This alternative is given by the legal usage of minority languages as languages of instruction and official languages beside the national language. The second level is the language policy of the state in the teaching of foreign languages. At this level the situation is quite unstable. There are periods of changes in short intervals and the question of further teacher training for younger school age children (primary education) remains unsolved.

\section{INDEX}

Keywords : foreign language, language policy, plurilingualism, teacher training

Mots-clés : formation des enseignants, langue étrangère, plurilinguisme, politiques

linguistiques

\section{AUTEUR}

\section{ANNA BUTAŠOVÁ}

Professeur, Université Comenius, Faculté de Pédagogie, Bratislava 